\title{
Vela, M. (2019). Correspondencia entre música y palabra. Un estudio sinestésico sobre Harmonio du soir, Baudelaire/Debussy y Le Gibet, Bertrand/Ravel. Vigo: Academia Editorial del Hispanismo
}

Hace varios siglos que música y palabra se juntaron para formar un fuerte lazo que se prolongaría durante años hasta la llegada de la música puramente instrumental. En aquel momento, la palabra era la protagonista y la música un mero acompañamiento de aquella. Pero esta relación no siempre ha sido así y ha sufrido cambios y modificaciones con el paso del tiempo. En esta obra, la autora expone una perspectiva distinta de la música a la que no estamos tan acostumbrados, puesto que está más centrada en la influencia que ha tenido la palabra sobre la música y cómo los compositores de cada época tuvieron que regirse por la estética musical del momento. Se comentan los elementos que ambas partes poseen y que hacen que puedan funcionar juntas, así como se analizan dos obras tanto literarias como musicales que están relacionadas entre sí por servirse de inspiración mutua.

\section{Breve perspectiva histórica de la relación entre música y palabra}

En este capítulo, la autora hace un interesante y bien fundamentado recorrido de fuentes primarias y secundarias que van desde los clásicos griegos hasta prácticamente el siglo XVII, para demostrar cómo la música era un soporte de la palabra, en especial en la música religiosa, debido a que el texto podía dotarla de significado. Pero no fue hasta finales del siglo XVI cuando la música se separó del texto y adoptó el contrapunto como una característica propia de la música instrumental, algo que la música vocal del momento había ya abandonado. Así, las distintas fuentes documentales permiten a la autora llegar a la conclusión de que, durante aproximadamente un siglo, la música se fue cimentando y aprendiendo de la música vocal y, en especial, de la ópera que fue la que más la influyó en su evolución.

En ese momento, los compositores sintieron que la música tenía que independizarse del texto y desarrollarse por sí misma: las corrientes románticas revolucionarían la música al afirmar que era la única vía de comunicación auténtica y sincera que trascendía de la palabra, que era más ambigua y confusa. En este sentido, se incluyen muchas fuentes que atestiguan fielmente esta afirmación romántica. Pero cuando parecía que la música instrumental era el mejor vehículo expresivo, estos compositores vieron que la música necesitaba de algo fuera de lo que le era característico para completarse: nuevamente, retornan a la necesidad del texto. De aquí surgió lo que se conocerá como música programática y que tuvo sus primeros pasos con Beethoven, como afirma la autora tras el análisis documental realizado.

@ARTURO JOSÉ CALAFORRA GUARDEÑO. THE CONTENT OF THIS ARTICLE IS THE SOLE RESPONSIBILITY OF THE AUTHORS. THE REVISTA ELECTRÓNICA DE LEEME AND UNIVERSITAT DE VALĖNCIA ARE NOT LIABLE FOR ANY LEGAL ACTIONS THAT MAY ARISE INVOLVING THE ARTICLE'S CONTENT. REVISTA ELECTRÓNICA DE LEEME - LISTA ELECTRÓNICA EUROPEA DE MÚSICA EN LA EDUCACIÓN-HTTP://OJS.UV.ES/INDEX/PHP/LEEME/INDEX. ISSN: 1575-9563. EDITORES: UNIVERSIDAD DE VALENCIA Y JESÚS TEJADA GIMÉNEZ. VISIBILIDAD DE ESTA REVISTA: SCOPUS, EMERGING SOURCES CITATION INDEX (CLARIVATE), EBSCO, CINDOC (CSIC), CITEFACTOR, COPAC, DIALNET, DICE (CSIC), DOAJ, E-REVISTAS (CSIC), EBSCO PREMIER, ERIH+, GALE CENGAGE LEARNING, IN-RECS, IRESIE, LATINDEX, MIAR, OCLC WORLDCAT, RESH, REDIB, RILM CORE JOURNALS, SUDOC, ULRICHS, ESTA REVISTA ESTÁ PUBLICADA CON EL APOYO INSTITUCIONAL DE REDIRIS-CONSEJO SUPERIOR DE INVESTIGACIONES CIENTIFICAS Y ES DE ACCESO LIBRE. CREATIVE COMMONS LICENSE 4.O BY 
La idea era reconciliar el texto con el acompañamiento instrumental, dando como resultado el lied de Schubert y las óperas de Wagner. Es más, sería Liszt, inspirado por la cultura francesa que buscaba con el racionalismo dar explicación a cualquier fenómeno, quien revolucionara el panorama sinfónico añadiendo un programa literario que explicara el contenido de la música y que culminaría con el nacimiento de un nuevo género: el poema sinfónico. En este sentido, la autora expone la idea de que lo que se pretendía era que el público pudiera entender las intenciones que tuvo el compositor a la hora de componer la obra. Berlioz con su Sinfonía fantástica junto con Listz fueron los que engendraron este nuevo género instrumental, teniendo su culmen en el leitmotive de Wagner. Aquí, la autora expone que esta unión de texto y música coincidió con el cambio social que hubo en Europa y con la idea de hacer que la música, ese arte misterioso y que estaba al alcance de unos pocos, pudiera llegar al máximo número de personas posibles y pudiera ser comprendida completamente por el público.

Llegados a este momento del capítulo, se señala que no todo fue tan idílico como se planteaba en los documentos de la época, ya que durante este resurgir de la música aunando fuerzas con el texto, hubo eruditos y artistas que se opusieron a esta nueva idea y aparecieron dos bandos: los músicos formalistas, que pensaban que la música era suficiente por sí misma y no necesitaba de elementos externos para ser tocada (Brahms); y los músicos contenidistas, que aceptaban esta nueva música programática. A todo esto, la autora señala las duras críticas hacia Wagner por parte de Hanslick, por ejemplo; aunque, curiosamente, después de su muerte se convirtió en fuente de inspiración para los nuevos compositores franceses y alemanes como objetivo de críticas. Con la llegada del siglo XX, este debate entre formalistas y contenidistas fue desapareciendo en pos de una nueva estética innovadora y que rompía con los parámetros tradicionales de la música.

\section{La cuestión entre la semanticidad}

El interés de este capítulo se centra en mostrar que tanto la música como el texto tienen en común el significado, aunque cada una la lleva de una forma distinta de la otra y esto hace que, al final, ambas intenten cubrirse los defectos con la otra. Pero, entonces, aquí se plantea un interrogante: ¿qué hace tan especial al texto y a la música que hace que se complementen? Aquí, la autora parte de las siguientes consideraciones: primero, el texto tiene una vía de comunicación que, si se usa de la forma más habitual y común, se puede utilizar para comunicarse con otros; pero, cuando se quiere utilizar un lenguaje más expresivo, como le ocurre a la poesía, la palabra es solo un lenguaje cotidiano que intenta transmitir un mensaje expresivo, más artístico, lo cual a la música no le ocurre, por eso el texto busca a la música. En segundo lugar, la música supone o se convierte en un lenguaje mucho más expresivo que el texto, pero carece de significado para que pueda ser entendido por todos, mientras que el texto sí dispone e intenta que la música se una fácilmente con el texto.

@ARTURO JOSÉ CALAFORRA GUARDEÑO. THE CONTENT OF THIS ARTICLE IS THE SOLE RESPONSIBILITY OF THE AUTHORS. THE REVISTA ELECTRÓNICA DE LEEME AND UNIVERSITAT DE VALĖNCIA ARE NOT LIABLE FOR ANY LEGAL ACTIONS THAT MAY ARISE INVOLVING THE ARTICLE'S CONTENT. REVISTA ELECTRÓNICA DE LEEME -LISTA ELECTRÓNICA EUROPEA DE MÚSICA EN LA EDUCACIÓN.HTTP://OJS.UV.ES/INDEX/PHP/LEEME/INDEX. ISSN: 1575-9563. EDITORES: UNIVERSIDAD DE VALENCIA Y JESÚS TEJADA GIMÉNEZ. VISIBILIDAD DE ESTA REVISTA: SCOPUS, EMERGING SOURCES CITATION INDEX (CLARIVATE), EBSCO, CINDOC (CSIC), CITEFACTOR, COPAC, DIALNET, DICE (CSIC), DOAJ, E-REVISTAS (CSIC), EBSCO PREMIER, ERIH+, GALE CENGAGE LEARNING, IN-RECS, IRESIE, LATINDEX, MIAR, OCLC WORLDCAT, RESH, REDIB, RILM CORE JOURNALS, SUDOC, ULRICHS, ESTA REVI
CON EL APOYO INSTITUCIONAL DE REDIRIS-CONSEJO SUPERIOR DE INVESTIGACIONES CIENTIFICAS Y ES DE ACCESO LIBRE. CREATIVE COMMONS LICENSE 4.O BY 
En el caso de la música, a raíz de lo comentado anteriormente por la autora, en el siglo XX se buscaba un intento de decodificación del significado de la música comparándolo con la poesía, pero era una tarea muy ardua de realizar ya que, a pesar de que la música tiene una jerarquización de unidades (notas con fonemas, motivos con palabras, etc.) que llegan a ser cada vez más complejas al igual que el texto, en realidad el sonido carece de una relación entre significante y significado, por lo que cualquier intento siempre llevaría al fracaso. Esto también es debido a que, si en algún momento se le diera a un elemento una relación sonora, solamente tendría sentido para el compositor que le ha querido dar esa relación; es decir, que tal como concluye la autora, cada compositor es único y su forma de trabajar es exclusiva de ese compositor, por lo que, al final, no se podría llegar a crear un sistema común que todos pudiéramos entender.

En este momento, el capítulo presenta opiniones de muchos autores con ideas muy contradictorias entre sí, lo que lleva al lector a considerar que no hay posiciones claras e iguales respecto a la posición que debería tener la música. Se nos muestra cómo algunos autores intentaron crear este sistema que dotaba a la música de significados particulares, pero pronto se dieron cuenta que no era efectivo, ya que solo pensaban en las relaciones interválicas de las notas y no en el ritmo. Otros, en cambio, piensan que esto no se debería dotar de significado expresivo a este tipo de intervalos musicales. Y otros piensan que la música realmente es un lenguaje porque, o bien se alejaba de la palabra y creaba su propio significado de carácter general y trascendente (que es así como pensaban los formalistas), o bien tiene las mismas relaciones que el lenguaje en cuanto a organización de los sonidos se refiere, aunque al final tenga siempre un significado ambiguo. Todo esto lleva a la autora a concluir este capítulo afirmando que lo más coherente es pensar que la música y el texto en vez de fusionarse tienen que hacer una trans-fusión entre ellas, entendiendo esto como que pueden cogerse elementos entre ellas y apoyarse una con la otra, dejando atrás las ideas de aunar ambas en una sola sin que predomine una sobre la otra, es decir, que ambas sean igualadas.

\section{Correspondencias: estructuras y procesos compartidos}

En este capítulo, la autora se centra en las características principales que tienen tanto la música como el texto y que hacen que su relación sea tan cercana; así, se centra en los siguientes conceptos que ha considerado clave:

En primer lugar, habla de la forma y ritmo estructural. Este componente es esencial tanto para la música como para el texto porque, según la forma y estructura que tenga la obra, la interpretación de la misma podrá ser de una manera u otra. Pone como ejemplo el Prélude à l'après-midi d'un faune escrita por Mallarmé y establece una comparación con la obra con el mismo título del compositor Debussy, comentando la estructura de ambas, la correlación que

@ARTURO JOSÉ CALAFORRA GUARDEÑO. THE CONTENT OF THIS ARTICLE IS THE SOLE RESPONSIBILITY OF THE AUTHORS. THE REVISTA ELECTRÓNICA DE LEEME AND UNIVERSITAT DE VALĖNCIA ARE NOT LIABLE FOR ANY LEGAL ACTIONS THAT MAY ARISE INVOLVING THE ARTICLE'S CONTENT. REVISTA ELECTRÓNICA DE LEEME -LISTA ELECTRÓNICA EUROPEA DE MÚSICA EN LA EDUCACIÓN.HTTP://OJS.UV.ES/INDEX/PHP/LEEME/INDEX. ISSN: 1575-9563. EDITORES: UNIVERSIDAD DE VALENCIA Y JESÚS TEJADA GIMÉNEZ. VISIBILIDAD DE ESTA REVISTA: SCOPUS, EMERGING SOURCES CITATION INDEX (CLARIVATE), EBSCO, CINDOC (CSIC), CITEFACTOR, COPAC, DIALNET, DICE (CSIC), DOAJ, E-REVISTAS (CSIC), EBSCO PREMIER, ERIH+, GALE CENGAGE LEARNING, IN-RECS, IRESIE, LATINDEX, MIAR, OCLC WORLDCAT, RESH, REDIB, RILM CORE JOURNALS, SUDOC, ULRICHS, ESTARE
CON EL APOYO INSTITUCIONAL DE REDIRIS-CONSEJO SUPERIOR DE INVESTIGACIONES CIENTIFICAS Y ES DE ACCESO LIBRE. CREATIVE COMMONS LICENSE 4.O BY 
tienen entre sí y algunos elementos extras como puede ser la utilización de la proporción áurea en ambos.

En segundo lugar, comenta acerca de la selección y combinación/repetición y variación: desarrollo temático. Este punto lo basa en la teoría de Jakobson sobre la proyección del eje paradigmático sobre el eje sintagmático en la función poética, quien comenta que existen dos elementos a tener en cuenta: la equivalencia y la combinación. El primer término trata de la relación semántica de dos elementos de una misma obra (sinónimo-antónimo), y el segundo consiste en la contigüidad de los elementos; aunque ambos elementos se pueden aplicar tanto a la poesía como a la música y son las encargadas de dar sentido a la estructura de la obra y de cohesión interna de la misma. Un buen ejemplo de todo esto, al menos referido a la música, sería la sonata clásica, la cual tiene una exposición y una reexposición que repite el mismo tema, y un desarrollo que modifica y varia los temas expuestos previamente, dándole sentido y coherencia a toda la obra. Existen muchos más ejemplos, pero en esta obra pone de ejemplo la Sinfonía fantástica de Berlioz y la compara con el programa que utilizó en el estreno de la misma, viéndose que en ambas obras gira entorno a un tema en concreto que se va desarrollando y variando; entre otros ejemplos que comenta la autora con cuidado detalle.

En tercer lugar, explicaría la organización sintáctica: armonía. Se podría decir que la función de la armonía en la música es la misma que la sintaxis al texto, es decir, una propiedad que hace que el texto pueda organizarse de forma correcta y que ésta tenga sentido y cohesión. Dentro de la armonía, existirían dos tipos de armonía: una más centrada en la estructura armónica, y otra que podría considerarse como adornos; al igual que se puede observar en la sintaxis del lenguaje.

En cuarto lugar, se centraría en la atmosfera: emoción e intuición. Es complicado llegar a traducir cierto contenido sonoro a palabras, por lo que la única forma de poder sentir lo que la música nos quiere transmitir alguna emoción es mediante la intuición. De modo que, la forma de crear una correlación entre ambas artes es crear, dentro de la obra musical, una atmosfera ambientada e inspirada por un texto; así, contendrá ambas intenciones y se podrá decir que intenta transmitir un mensaje menos ambiguo, juntando las mejores partes de la música y el texto. Como ejemplos, la autora comenta algunas obras de Schubert, Listz, Beethoven, Schumann y Tchaikovsky, siempre contextualizando con los poemas o textos que se han inspirado los compositores a la hora de crear sus obras.

Por último, termina hablando de los recursos descriptivos. Este punto, aunque muy breve, recuerda que existen recursos descriptivos que hacen que la obra adquiera mucha más riqueza y se pueda captar la idea que tenía el compositor-escritor a la hora de realizarla. Se comenta la Sonata $\mathrm{n}^{\circ}$ 81a de Beehoven y su forma de conseguir que el piano recuerde al sonido de una trompa y adquiera cierto significado extra; un lied de Schubert inspirado en un poema de Müller titulado Das Leiermann (el organillero) que imita este instrumento tocado por un músico apartado de la

@ARTURO JOSÉ CALAFORRA GUARDEÑO. THE CONTENT OF THIS ARTICLE IS THE SOLE RESPONSIBILITY OF THE AUTHORS. THE REVISTA ELECTRÓNICA DE LEEME AND UNIVERSITAT DE VALĖNCIA ARE NOT LIABLE FOR ANY LEGAL ACTIONS THAT MAY ARISE INVOLVING THE ARTICLE'S CONTENT. REVISTA ELECTRÓNICA DE LEEME - LISTA ELECTRÓNICA EUROPEA DE MÚSICA EN LA EDUCACIÓN-HTTP://OJS.UV.ES/INDEX/PHP/LEEME/INDEX. ISSN: 1575-9563. EDITORES: UNIVERSIDAD DE VALENCIA Y JESÚS TEJADA GIMÉNEZ. VISIBILIDAD DE ESTA REVISTA: SCOPUS, EMERGING SOURCES CITATION INDEX (CLARIVATE), EBSCO, CINDOC (CSIC), CITEFACTOR, COPAC, DIALNET, DICE (CSIC), DOAJ, E-REVISTAS (CSIC), EBSCO PREMIER, ERIH+, GALE CENGAGE LEARNING, IN-RECS, IRESIE, LATINDEX, MIAR, OCLC WORLDCAT, RESH, REDIB, RILM CORE JOURNALS, SUDOC, ULRICHS, ESTA REVISTA ESTÁ PUBLICADA CON EL APOYO INSTITUCIONAL DE REDIRIS-CONSEJO SUPERIOR DE INVESTIGACIONES CIENTIFICAS Y ES DE ACCESO LIBRE. CREATIVE COMMONS LICENSE 4.O BY 
sociedad; en Première année de pèlerinage: Suisse de Listz que describe con la música una tormenta en un lago; y la descripción de Debussy de Puck en la obra El sueño en una noche de verano de Shakespeare.

Teniendo más claros cada punto a tener en cuenta, se podrá entender mejor los análisis que la autora realiza en el siguiente capítulo, donde ya se va ahondando con mayor profundidad en el meollo de esta obra.

\section{La sinestesia como símbolo común}

Los autores que se comentan en este capítulo son franceses, esta selección tiene su explicación en el hecho de que, en el siglo XIX, Francia fue el país donde la unión entre poesía y música se hizo más fuerte, llegando a influenciar al resto de países, tales como Alemania. Los compositores comentados principalmente son Debussy y Ravel, quienes se inspiraron en alguna de las obras de Baudelaire y Bertrand para crear sus composiciones.

En este capítulo, la autora comienza a explicar el texto Harmonie du soir, del poemario titulado Les Fleurs du mal, de Baudelaire. A modo de resumen, se comenta el contexto donde nació esta obra y un poco de dónde vino la inspiración de Debussy para crear la composición que luego se comenta; se analiza el tipo de estrofa y rimas que tiene el poema, siendo la estrofa de tipo alejandrino con una estructura cíclica con rimas en la primera y cuarta estrofa y en la segunda y tercera; y el tema gira en torno a la vida y la muerte, siendo éstas representadas por tige (tallo, que alimenta a la planta) y soir (tarde, simbolizando la muerte); entre otros elementos que son más especificados por la autora en dicho capítulo: la semanticidad de cada párrafo, el clímax del poema, el ritmo estructural, etc.

La siguiente obra que se ha seleccionado es la obra para piano Les sons et les parfums se tournent dans l'air du soir, el primer preludio de los doce que contiene el volumen compuesto por Debussy. Esta obra, influida por la obra de Baudelaire ya comentada por la autora, tiene una estructura basada en la proporción áurea y en la sucesión de Fibonacci y que es retrogradable. Tiene 3 temas principales, A, B y C, que se distribuyen de forma que se asemeja a una forma rondó, pero sin serlo, siendo el orden A1, B, A2, C, A3, A4, guardando similitud con el poema de Baudelaire en cuanto a los temas empleados, la tensión, el ritmo de la obra, entre otros muchos factores. Además de analizar cada sección de la obra detenidamente y aportar las ideas e intenciones que tuvo el compositor a la hora de realizar su creación, deja un último apartado donde explica con más detalle los puntos en común entre ambas obras.

El otro poema que se comenta en el libro es Le Gibet del poemario Gaspard de la nuit, de Bertrand. La autora señala que este poema tiene de especial que está escrito en prosa, es de temática fantástica situada en el Medievo y donde presenta a Gaspard de la nuit, un personaje misterioso relacionado con Satán. Lo que más destaca son las preguntas retóricas que

@ARTURO JOSÉ CALAFORRA GUARDEÑO. THE CONTENT OF THIS ARTICLE IS THE SOLE RESPONSIBILITY OF THE AUTHORS. THE REVISTA ELECTRÓNICA DE LEEME AND UNIVERSITAT DE VALĖNCIA ARE NOT LIABLE FOR ANY LEGAL ACTIONS THAT MAY ARISE INVOLVING THE ARTICLE'S CONTENT. REVISTA ELECTRÓNICA DE LEEME - LISTA ELECTRÓNICA EUROPEA DE MÚSICA EN LA EDUCACIÓN-HTTP://OJS.UV.ES/INDEX/PHP/LEEME/INDEX. ISSN: 1575-9563. EDITORES: UNIVERSIDAD DE VALENCIA Y JESÚS TEJADA GIMÉNEZ. VISIBILIDAD DE ESTA REVISTA: SCOPUS, EMERGING SOURCES CITATION INDEX (CLARIVATE), EBSCO, CINDOC (CSIC), CITEFACTOR, COPAC, DIALNET, DICE (CSIC), DOAJ, E-REVISTAS (CSIC), EBSCO PREMIER, ERIH+, GALE CENGAGE LEARNING, IN-RECS, IRESIE, LATINDEX, MIAR, OCLC WORLDCAT, RESH, REDIB, RILM CORE JOURNALS, SUDOC, ULRICHS, ESTA REVISTA ESTÁ PUBLICADA CON EL APOYO INSTITUCIONAL DE REDIRIS-CONSEJO SUPERIOR DE INVESTIGACIONES CIENTIFICAS Y ES DE ACCESO LIBRE. CREATIVE COMMONS LICENSE 4.O BY 
constantemente va haciendo y que hacen dudar qué es realidad y qué es fantasía; todas estas cuestiones serán resueltas al final del texto con una última pregunta. Tiene una estructura sintáctica reiterativa, puesto que las preguntas están enlazadas unas con otras. Estos y muchos más elementos son comentados con gran detalle durante este capítulo.

Por último, la obra de Ravel titulada igual que el poema de Bertrand, llegando a asemejarse al poema, por ejemplo, en la estructura ternaria (A, B, A') y cíclica, creada a partir de la sección áurea. Ravel intenta recrear con la música el poema de Bertrand, haciendo uso de recursos descriptivos como imitar el sonido de una campana. Entre otros elementos que se explican, el que más destaca es el uso del que se llama el acorde de Tristán, utilizado por Wagner como leitmotiv del deseo, que lo utiliza a lo largo de la obra. Para terminar, y al igual que ha pasado con la comparación entre Baudelaire y Debussy, la autora aprovecha para explicar con más detalle los elementos en común en el poema y en la obra musical descritas.

La autora incluye unos completos Anexos con las obras musicales analizadas al detalle, donde los músicos más teóricos interesados en el análisis formal, armónico y contrapuntístico, podrán conocer con más detalle las obras analizadas en este libro. Pero, lo más importante de esta obra quizá sea el recordar a los músicos (intérpretes, teóricos y oyentes) la importancia de la palabra en la música y de la música en la palabra, como complementarias a la par que no excluyentes.

\author{
Autor de la recensión \\ Arturo José Calaforra Guardeño \\ arcaguar@alumni.uv.es
}

@ARTURO JOSÉ CALAFORRA GUARDEÑO. THE CONTENT OF THIS ARTICLE IS THE SOLE RESPONSIBILITY OF THE AUTHORS. THE REVISTA ELECTRÓNICA DE LEEME AND UNIVERSITAT DE VALĖNCIA ARE NOT LIABLE FOR ANY LEGAL ACTIONS THAT MAY ARISE INVOLVING THE ARTICLE'S CONTENT. REVISTA ELECTRÓNICA DE LEEME -LISTA ELECTRÓNICA EUROPEA DE MÚSICA EN LA EDUCACIÓN.HTTP://OJS.UV.ES/INDEX/PHP/LEEME/INDEX. ISSN: 1575-9563. EDITORES: UNIVERSIDAD DE VALENCIA Y JESÚS TEJADA GIMÉNEZ. VISIBILIDAD DE ESTA REVISTA: SCOPUS, EMERGING SOURCES CITATION INDEX (CLARIVATE), EBSCO, CINDOC (CSIC), CITEFACTOR, COPAC, DIALNET, DICE (CSIC), DOAJ, E-REVISTAS (CSIC), EBSCO CON EL APOYO INSTITUCIONAL DE REDIRIS-CONSEJO SUPERIOR DE INVESTIGACIONES CIENTIFICAS Y ES DE ACCESO LIBRE. CREATIVE COMMONS LICENSE 4.O BY 